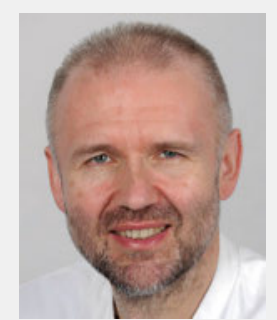

Prof. Dr. med. Wolfgang Hartl

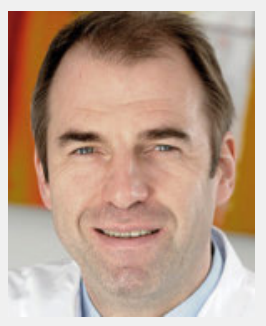

Prof. Dr. med. Jens Werner

\section{Enterale vs. parenterale Ernährung: Wie groß sind die Unterschiede wirklich?}

Viele kritisch kranke Patienten entwickeln als Folge einer prolongierten schweren Infektion oder Inflammation eine Atrophie ihrer Skelettmuskulatur bzw. eine muskuläre Schwäche. Eine effektive Prophylaxe dieser Veränderungen ist nur durch die kausale Therapie der auslösenden Grunderkrankung (z. B. Peritonitis, hämorrhagischer Schock) möglich. Die Ernährungstherapie kann als adjuvante Therapie helfen, entsprechende Veränderungen abzumildern.

Kritisch kranke Patienten benötigen im Rahmen ihrer Ernährungstherapie eine regelmäßige Kalorienzufuhr. In der Vergangenheit wurde aus verschiedenen pathophysiologischen Überlegungen heraus empfohlen, eine enterale (gastrale/jejunale) Ernährung gegenüber der parenteralen (i.d. R. zentralvenösen Ernährung) zu bevorzugen [1-3]. Aus klinischer und tierexperimenteller Sicht beruhte diese Empfehlung darauf, dass im Vergleich zur parenteralen Ernährung eine enterale Ernährung mit einer geringeren Infektionsrate, einer verbesserten Integrität der Darmmukosa und mit mutmaßlich geringeren Kosten verbunden war [4]. Kritikpunkt war jedoch bisher, dass die Nachteile einer parenteralen Ernährung möglicherweise nicht auf dem Zugangsweg an sich beruhten, sondern speziell darauf, dass dieser Weg der Nahrungszufuhr häufig mit einer Überernährung (v. a. einer exzessiven Zufuhr von Glukose) verbunden war. Bis vor Kurzem gab es keine Studie, die bei vergleichbarer Zufuhr von Kalorien (speziell Kohlenhydrate) und bei einer vergleichbaren Funktion des Gastrointestinaltrakts ausschließlich die verschiedenen Wege der Nahrungszufuhr (enteral vs. parenteral) miteinander verglichen hätte. Diese Lücke wurde jetzt durch die CALORIES-Studie geschlossen [5].

In dieser großen multizentrischen prospektiven randomisierten Studie wurden 2400 erwachsene kritisch kranke Patienten, die sowohl enteral wie auch parenteral hätten ernährt werden können, hinsichtlich dieser beiden Wege der Nahrungszufuhr randomisiert. Die Kalorien- und Eiweißzufuhr war dabei in beiden Studienarmen miteinander vergleichbar. Zentrales Ergebnis der Studie war, dass der Zugangsweg ohne Auswirkung auf die 30-Tages-Letalität war. Auch sekundäre Endpunkte (Häufigkeit von Infektionen, Ausmaß des Organversagens) blieben - mit Ausnahme von mehr Erbrechen unter enteraler Ernährung - unbeeinflusst.

Diese Ergebnisse decken sich mit den Resultaten einer kürzlich publizierten Metaanalyse [6], die vergleichende Studien zur enteralen/parenteralen Ernährung auswertete. Unabhängig von der Höhe der Kalorienzufuhr bestand hinsichtlich der Letalität kein Unterschied zwischen enteraler und parenteraler Ernährung. Eine erhöhte Morbidität (vermehrte Rate an Infektionen) konnte nur dann beobachtet werden, wenn die Patienten unter parenteraler Ernährung auch gleichzeitig mehr Kalorien bekommen hatten. Die für die parenterale Ernährung ungünstigen Ergebnisse waren darüber hinaus nur bei Studien zu beobachten, die älter als 20 Jahre waren und eine ungenügende Kontrolle der Blutzuckerkonzentration aufwiesen. Zusätzlich bestand ein substanzieller Publikations-Bias.

Somit scheint es unter Zufuhr von gleichen Mengen an Kalorien und Stickstoff in der klinischen Praxis keine relevanten Unterschiede zwischen enteraler und parenteraler Ernährung zu geben. Eine Kosten-Nutzen-Analyse der CALORIES-Studie konnte jedoch eine deutliche Kostenersparnis unter enteraler Ernährung zeigen [7]. Somit sollte - sofern keine Kontraindikationen bestehen - die enterale Ernährung bevorzugt werden, wobei jedoch kein Zwang zur aggressiven Implementierung dieses Weges der Nahrungszufuhr besteht. 
[1] Singer P, Berger MM, Van den Berghe $G$ et al. ESPEN guidelines on parenteral nutrition: intensive care. Clin Nutr 2009; 28: 387-400

[2] McClave SA, Martindale RG, Vanek VW et al. Guidelines for the provision and assessment of nutrition support therapy in the adult critically ill patient: Society of Critical Care Medicine (SCCM) and American Society for Parenteral and Enteral Nutrition (A.S.P.E.N.). JPEN J Parenter Enteral Nutr 2009; 33: 277-316

[3] McClave SA, Taylor BE, Martindale RG et al. Guidelines for the provision and assessment of nutrition support therapy in the adult critically ill patient: Society of Critical Care Medicine (SCCM) and American Society for Parenteral and Enteral Nutrition (A.S.P.E.N.). JPEN J Parenter Enteral Nutr 2016; 40: 159-211

[4] Casaer MP, Van den Berghe G. Nutrition in the acute phase of critical illness. N Engl J Med 2014; 370:1227-1236

[5] Harvey SE, Parrott F, Harrison DA et al. CALORIES Trial Investigators. Trial of the route of early nutritional support in critically ill adults. N Engl J Med 2014; 371: 1673-1684

[6] Elke G, van Zanten AR, Lemieux M et al. Enteral versus parenteral nutrition in critically ill patients: an updated systematic review and meta-analysis of randomized controlled trials. Crit Care 2016; 20: 117

[7] Harvey SE, Parrott F, Harrison DA et al. A multicentre, randomised controlled trial comparing the clinical effectiveness and cost-effectiveness of early nutritional support via the parenteral versus the enteral route in critically ill patients (CALORIES). Health Technol Assess 2016; 20: 1-144 